\title{
The bahasa Melayu version of cornell musculoskeletal discomfort questionnaire (CMDQ): reliability and validity study in Malaysia
}

\begin{abstract}
BACKGROUND: The Cornell Musculoskeletal Discomfort Questionnaire (CMDQ) was developed to assess the level of musculoskeletal discomfort among office workers related to their ergonomic situation. OBJECTIVE: The primary objective of this initial study is to analyze the validity and dependability of the Malay translation of the Cornell Musculoskeletal Discomfort Questionnaire. METHODS: The questionnaire was selfadministered two times, with an interval of two weeks in order to evaluate the accuracy of the original findings with a retest. The study involved 115 participants. RESULTS: The range of Cronbach Alpha coefficient showed a considerable consistency of the items for each subscale (Cronbach's a $>0.95$ ). The range of Kappa coefficients was between (ICC $=0.690$ $0.949, \mathrm{p}<0.001), \quad(\mathrm{ICC}=0.801-0.979, \mathrm{p}<0.001)$ and $(\mathrm{ICC}=0.778-0.944, \mathrm{p}<0.001)$ for frequency, severity and interference scales. CONCLUSIONS: This research, introduced the Malay-language version of the CMDQ (CMDQ-M) as the first formal validation of the CMDQ, and confirmed a high reliability and validity for the evaluation of musculoskeletal discomfort among the study population.
\end{abstract}

Keyword: Office ergonomics; Musculoskeletal disorders; Physical conditioning; Anatomy 